\title{
Estimación de excreción fecal en alpacas (Vicugna pacos) bajo condiciones estabuladas usando dióxido de titanio
}

\author{
Estimation of faecal excretion in alpacas (Vicugna pacos) in confinement \\ conditions using titanium dioxide
}

\author{
Rosina Camargo H. ${ }^{1}$, Carlos Vílchez P. ${ }^{2}$, Juan Olazábal L. ${ }^{1}$, Felipe San Martín H. ${ }^{1}$
}

\section{Resumen}

\begin{abstract}
El objetivo del estudio fue estimar la producción de heces en alpacas con el uso del dióxido de titanio $\left(\mathrm{TiO}_{2}\right)$. Se usó un diseño cuadrado latino $4 \mathrm{x} 4$, con cuatro animales, cuatro tratamientos y cuatro periodos. Cada periodo de 14 días de experimentación y siete de descanso. Los tratamientos fueron: T2.5(1), $2.5 \mathrm{~g}$ de $\mathrm{TiO}_{2} /$ animal/día; $\mathrm{T} 1.25(2), 2.5 \mathrm{~g}$ de $\mathrm{TiO}_{2} /$ animal/día repartido en dos dosis (07:00, 16:00); T5(1), $5 \mathrm{~g}$ de $\mathrm{TiO}_{2}$ /animal/día; T2.5(2), $5 \mathrm{~g}$ de $\mathrm{TiO}_{2} /$ animal/día repartido en dos dosis (07:00, 16:00). Los porcentajes de la recuperación de $\mathrm{TiO}_{2}$ fueron de $90,82,78$ y 52\% para T2.5(1), T1.25(2), T5(1) y T2.5(2), respectivamente, siendo T2.5(2) diferente a los otros tratamientos $(\mathrm{p}=0.00013)$. Los parámetros del patrón de excreción del $\mathrm{TiO}_{2}$ fueron: intercepto de la curva de la excreción de $\mathrm{TiO}_{2}$ en el tiempo cero (a), máxima excreción del $\mathrm{TiO}_{2}$ en tiempo (b), la tasa constante de excreción de b (c), tiempo necesario de excreción de $\mathrm{TiO}_{2}$ para que haya excretado la mitad de la concentración inicial $\left(\mathrm{T}_{1 / 2}\right)$ y tiempo para alcanzar el equilibrio de excreción del marcador $\left(\mathrm{T}_{(\mathrm{a}+\mathrm{b}}\right)$. No hubo diferencia entre tratamientos para los valores de a, c, T medio y $\mathrm{T}$, pero hubo diferencia significativa del valor de b entre la administración del marcador de 2.5 vs. $5 \mathrm{~g}(\mathrm{p}<0.0001)$. Los promedios de producción de heces estimada con $\mathrm{TiO}_{2}$ vs. producción de heces observada directamente fue diferente en $\mathrm{T} 1.25(2), \mathrm{T} 5(1)$ y T2.5(2) $(\mathrm{p}<0.05)$, pero no en T2.5(1). En conclusión, el tiempo en el que se alcanza el equilibrio de excreción del $\mathrm{TiO}_{2}$ es de aproximadamente ocho días y la dosis de $2.5 \mathrm{~g}$ de $\mathrm{TiO}_{2}$ una vez al día estima con mayor precisión la producción fecal en alpacas.
\end{abstract}

Palabras clave: dióxido de titanio; alpacas; producción de heces; marcador externo

\footnotetext{
${ }^{1}$ Laboratorio de Bioquímica, Nutrición y Alimentación Animal, Facultad de Medicina Veterinaria, Universidad Nacional Mayor de San Marcos, Lima, Perú

${ }^{2}$ Departamento de Nutrición, Facultad de Zootecnia, Universidad Nacional Agraria La Molina, Lima, Perú

${ }^{3}$ E-mail: rosina.camargo@unmsm.edu.pe
} 
The aim of this study was to estimate the production of faeces in alpacas with the use of titanium dioxide (TiO2). A square $4 \times 4$ Latin design was used, with four animals, four treatments and four periods. Each period last for 14 days of evaluation and seven days of rest. The treatments were: T2.5 (1), $2.5 \mathrm{~g}$ of TiO$/$ /animal/day; $\mathrm{T} 1.25(2), 2.5 \mathrm{~g}$ of $\mathrm{TiO}_{2}$ /animal/day divided into two doses (07:00, 16:00); T5(1), $5 \mathrm{~g}$ of $\mathrm{TiO}_{2} /$ animal/day; $\mathrm{T} 2.5(2), 5 \mathrm{~g}$ of $\mathrm{TiO}_{2} / \mathrm{animal} /$ day divided into two doses $(07: 00,16: 00)$. The percentages of the recovery of $\mathrm{TiO}_{2}$ were $90,82,78$ and $52 \%$ for $\mathrm{T} 2.5(1), \mathrm{T} 1.25(2), \mathrm{T} 5(1)$ and $\mathrm{T} 2.5(2)$, respectively, being T2.5(2) different from the other treatments $(\mathrm{p}=0.00013)$. The parameters of the $\mathrm{TiO}_{2}$ excretion pattern were: intercept of the curve of $\mathrm{TiO}_{2}$ excretion at time zero (a), maximum excretion of $\mathrm{TiO}_{2}$ at time (b), constant rate of excretion of b (c), time needed of excretion of $\mathrm{TiO}_{2}$ so that it has excreted half of the initial concentration $\left(\mathrm{T}_{1 / 2}\right)$, and time to reach the balance of excretion of the marker $\left(\mathrm{T}_{(\mathrm{a}+\mathrm{b})}\right)$. There was no difference between treatments for the values of a, c, mean $\mathrm{T}$ and $\mathrm{T}$, but there was a significant difference in the value of $b$ between the administration of the marker of 2.5 vs $5 \mathrm{~g}(\mathrm{p}<0.0001)$. The averages of faecal production estimated with $\mathrm{TiO}_{2}$ vs. faecal production observed directly was different in T1.25(2), T5(1) and T2.5(2) ( $<<0.05)$, but not in T2.5(1). In conclusion, the time in which the excretion equilibrium of $\mathrm{TiO}_{2}$ is reached is approximately eight days and the dose of $2.5 \mathrm{~g}$ of $\mathrm{TiO}_{2}$ once a day more accurately estimates faecal production in alpacas.

Key words: titanium dioxide; alpacas; faecal production; external marker

\section{INTRODUCCIÓN}

Los camélidos sudamericanos (CSA), en especial la alpaca (Vicugna pacos) tiene importancia económica y social para el poblador altoandino. En el Perú, la masa ganadera de alpacas es de 3 millones 800 mil, que representa el $87 \%$ de la población de alpacas del mundo (MINAGRI, 2017).

La medición del consumo en animales al pastoreo es de suma importancia, ya que con esta información se puede estimar la soportabilidad de la pradera y el estado nutricional de los animales que la pastorean, permitiendo un mejor manejo técnico de las praderas y de la crianza animal (Forbes y Kyriazakis, 1995; McDonald y Greenhalgh, 1999). Esta información en praderas altoandinas es escasa, más aún en CSA, que dependen exclusivamente de estas praderas para su alimentación y poseen menores consumos comparativos con otras especies (San Martín y Van Saun, 2014).
Se han desarrollado diversos métodos para determinar el consumo; sin embargo, la medición directa y el método estándar de oro para estimar el consumo de alimentos en animales al pastoreo no es aún posible (Hodgson, 2004). Se han evaluado numerosos métodos indirectos para su estimación, entre ellos, uno de los métodos que estima valores confiables de consumo es el de la relación de la producción fecal diaria sobre la indigestibilidad del alimento (H/Digestibilidad-1) (Haro, 2012 y Hellwing et al., 2015).

La relación de la producción fecal diaria sobre la indigestibilidad del alimento obliga la estimación de la producción de heces. Esta medición puede ser directa mediante la colocación de bolsas colectoras al cuerpo de los animales por medio de arneses; sin embargo, esta medición tiene inconvenientes que podría dar lugar a pérdida de heces e influenciar en el comportamiento normal del consumo de los animales (Mayes y Dove, 2000). Para evitar el uso de estas bolsas y sus in- 
convenientes, se aplican técnicas indirectas con el uso de materiales indigeribles, tanto orgánicos e inorgánicos, a los que se les denomina «marcadores». El uso de marcadores externos (elementos inertes) son seguros y poco laboriosos, cuya concentración es determinada en solo una muestra fecal (Greenhalgh, 1982).

El marcador inerte más usado para estimar el volumen total de excreción fecal en rumiantes al pastoreo ha sido el óxido crómico $\left(\mathrm{Cr}_{2} \mathrm{O}_{3}\right)$. Sin embargo, su colección varia grandemente entre animales y, además, posee propiedades cancerígenas cuando es inhalado por los que manipulan el material (Myers et al., 2004). Una alternativa al $\mathrm{Cr}_{2} \mathrm{O}_{3}$ es el dióxido de titanio $\left(\mathrm{TiO}_{2}\right)$, que presenta menos variabilidad entre animales para la estimación de producción fecal y no tiene efectos negativos para la salud ni el medio ambiente. Además, el 100\% de $\mathrm{TiO}_{2}$ se excreta en las heces y es compatible con las mucosas y la piel (Titgemeyer, 1997).

Por las razones señaladas se planteó el presente estudio, con el fin de evaluar el uso del $\mathrm{TiO}_{2}$ en alpacas para la estimación de la producción fecal total. Para tal fin, los objetivos del estudio fueron determinar la dosis y frecuencia de administración, así como estimar el tiempo de recojo del $\mathrm{TiO}_{2}$ como marcador externo para estimar la excreción fecal en alpacas.

\section{Materiales y Métodos}

\section{Lugar de Ejecución y Animales}

El estudio se realizó en el Laboratorio de Bioquímica, Nutrición y Alimentación Animal de la Facultad de Medicina Veterinaria de la Universidad Nacional Mayor de San Marcos, Lima. Se utilizaron cuatro alpacas Huacaya machos adultos, en aparente buen estado de salud. Los animales se colocaron en jaulas individuales, suministrándoles alimento y agua ad libitum. El alimento utiliza- do simuló las características químiconutricionales de las pasturas naturales y estuvo compuesto de afrecho de trigo, harinilla de cebada, alfalfa molida y un suplemento mineral, con un contenido nutricional de $13 \%$ de proteína cruda, $25 \%$ de fibra cruda y 4.13 $\mathrm{Mcal} / \mathrm{kg}$ de energía bruta.

\section{Diseño Experimental}

El estudio se llevó a cabo con un diseño cuadrado latino de $4 \times 4$, con cuatro animales, cuatro tratamientos y cuatro periodos, con una duración de 14 días por periodo y siete días de descanso entre periodos. Los animales pasaron por un periodo de siete días de adaptación a la permanencia en las jaulas individuales, al manejo de la dosificación de cápsulas de gelatina vacías con el uso de lanza bolos y a la colocación y permanencia de bolsas colectoras de heces por arneses. La toma de muestras de heces $(200 \mathrm{~g})$ para la determinación de $\mathrm{TiO}_{2}$ se hizo a las 48, 96, $144,192,240,288$ y 336 horas del inicio de la administración del marcador. Las muestras fueron colectadas a las 07:00. Así mismo, se hizo la medición directa de la producción total de heces en las bolsas colectoras.

\section{Tratamientos}

Se trabajó con cuatro tratamientos: T2.5(1) y T5(1) recibió 2.5 y 5.0 g de $\mathrm{TiO}_{2} /$ animal/día a las 07:00, respectivamente, mientras que T1.25(2) y T2.5(2) recibió 2.5 y $5.0 \mathrm{~g}$ de $\mathrm{TiO}_{2} /$ animal/día distribuido en dos dosis (07:00 y 16:00) para lograr una concentración homogénea del marcador en el tracto digestivo. Se utilizó una fuente comercial de $\mathrm{TiO}_{2}\left(\mathrm{Merck}^{\circledR}\right)$. La dosificación se realizó durante los 14 días del periodo experimental.

\section{Análisis de Laboratorio}

La curva de calibración se realizó con heces de alpacas antes de la administración del marcador. Se tomaron seis muestras de $0.5 \mathrm{~g}$, las cuales luego de ser secadas y molidas, se les agregó 0, 0.002, 0.004, 0.006, 0.008 
y $0.01 \mathrm{~g} \mathrm{de} \mathrm{TiO}_{2}$, luego se analizaron mediante el método de cenizas secas, según el protocolo establecido por Ohmori et al. (2013). Las muestras de heces fueron analizadas por triplicado. El análisis obtuvo un coeficiente de correlación de 0.9929 y el modelo de la ecuación lineal fue $Y=162.8 X+0.001$. En forma similar, la concentración de $\mathrm{TiO}_{2}$ en las muestras de heces del estudio se analizaron con el mismo método.

La tasa de excreción del marcador se estimó con el modelo matemático de regresión no lineal propuesta para estudios de degradabilidad por Ørskov y McDonald (1979): $\mathrm{p}=\mathrm{a}+\mathrm{b}\left(1-\mathrm{e}^{\mathrm{ct}}\right)$, donde $\mathrm{p}$ : excreción estimada del $\mathrm{TiO}_{2}$ en el día $\mathrm{t}$, a: intercepto de la curva de la excreción de $\mathrm{TiO}_{2}$ en el tiempo cero, b: máxima excreción del $\mathrm{TiO}_{2}$ en tiempo, c: tasa de excreción del $\mathrm{TiO}_{2}$ o constante $\mathrm{K}$. Así mismo, $\mathrm{T}_{(\mathrm{a}+\mathrm{b})}$ es el tiempo en el cual se alcanza el equilibrio constante de excreción del $\mathrm{TiO}_{2}$.

El tiempo de vida media $\left(\mathrm{T}_{1 / 2}\right)$ es el tiempo que trascurre para que haya excretado la mitad de la concentración inicial de $\mathrm{TiO}_{2}$. Se estima con la expresión $\operatorname{Ln}(2) / K$. La estimación de producción de heces se determinó mediante la ecuación propuesta por Prigge et al. (1981): Excreción de heces en materia seca $(\mathrm{g} /$ día $)=($ marcador suministrado, $\mathrm{g} /$ día $)$ / (concentración del marcador en las heces, $\mathrm{g} / \mathrm{kg}$ de materia seca)

\section{Análisis Estadístico}

Los parámetros de excreción del marcador se analizaron mediante el análisis de varianza y se empleó la prueba de Duncan para determinar diferencias entre promedios; con un nivel de significancia de $5 \%$. Se utilizó el programa estadístico SAS v. 9.4.

La estimación de la producción de heces con el $\mathrm{TiO}_{2}$ y producción directa de heces con bolsas colectoras se analizó con la prueba de $t$ de Student con un nivel de significancia de $5 \%$ y regresión lineal.

\section{Resultados y Discusión}

\section{Recuperación del $\mathrm{TiO}_{2}$}

El porcentaje de la recuperación del $\mathrm{TiO}_{2}$ se presenta en el Cuadro 1. La mayor recuperación del marcador se logró en los tratamientos T2.5(1), T1.25(2) y T5(1), no habiendo diferencia significativa entre estos

En estudios con bovinos y ovinos al pastoreo se reportaron recuperaciones de $\mathrm{TiO}_{2}$ de prácticamente el 100\% (Hafez et al., 1988; Titgemeyer et al., 2001; Glindemann et al., 2009), mientras que estudios con aves se reporta una recuperación de $97.5 \%$ (Peddie et al., 1982) y en ratas de $98 \%$ (Njaa, 1961). En animales con dietas a base de forrajes se observaron las mayores diferencias de recuperación de $\mathrm{TiO}_{2}$ con un rango de variación de 79 a $125 \%$ (Titgemeyer et al., 2001).

Estudios con aves rapaces se obtuvieron recuperaciones bajas de $\mathrm{TiO}_{2}$ de $78-52 \%$ (Barton y Houston, 1991), cuyos valores son cercanos a lo reportado en el presente estudio. Las posibles causas de los bajos porcentajes de recuperación del marcador serían recuperaciones incompletas en las heces, que el marcador este saliendo como producto de desecho por la orina, que el marcador tome más tiempo de lo esperado para quedar en equilibrio en esta especie, o que el marcador pasa a través del tracto gastrointestinal en pulsos concentrados y se haya tomado las muestras en el momento en que los marcadores estuvieron en bajas concentraciones. Así mismo, es posible que el marcador tenga una mayor retención en el tracto digestivo (San Martín y Van Saun, 2014), aunque no necesariamente sea absorbido, o por pérdidas de muestra durante la manipulación como en el molido, la incineración y la disolución (Barton y Houston, 1991).

El criterio para ser considerado marcador nutricional ideal de un marcador es que la recuperación debe ser cerca al 100\% 
Cuadro 1. Recuperación del dióxido de titanio - $\mathrm{TiO}_{2}(\%)$ en heces de alpacas

\begin{tabular}{ccccc}
\hline \multirow{2}{*}{ Días } & \multicolumn{4}{c}{ Tratamientos $^{1}$} \\
\cline { 2 - 5 } & $2.5(1)$ & $1.25(2)$ & $5(1)$ & $2.5(2)$ \\
\hline 2 & 77 & 82 & 83 & 44 \\
4 & 74 & 82 & 72 & 54 \\
6 & 99 & 86 & 125 & 47 \\
8 & 82 & 82 & 73 & 48 \\
10 & 97 & 73 & 72 & 53 \\
12 & 83 & 88 & 73 & 62 \\
14 & 107 & 83 & 53 & 46 \\
\hline Promedio & $90 \pm 13^{\mathrm{a}}$ & $82 \pm 5^{\mathrm{a}}$ & $78 \pm 22^{\mathrm{a}}$ & $52 \pm 6^{\mathrm{b}}$ \\
\hline
\end{tabular}

a,b Superíndices diferentes dentro de filas indican diferencia significativa $(p<0.05)$

${ }^{1} \mathrm{~T} 2.5(1)$ y T5(1) recibieron 2.5 y $5.0 \mathrm{~g}$ de TiO2/animal/día a las 07:00, respectivamente. T1.25(2) y $\mathrm{T} 2.5(2)$ recibieron 2.5 y $5.0 \mathrm{~g}$ de TiO2/animal/día distribuido en dos dosis a las 07:00 y 16:00

Cuadro 2. Parámetros (promedio $\pm \mathrm{DE}$ ) del patrón de excreción de dióxido de titanio $\left(\mathrm{TiO}_{2}\right)$ en heces de alpaca

\begin{tabular}{cccccc}
\hline Tratamiento $^{1}$ & $\mathrm{a}\left(\mathrm{g} \times 10^{-6}\right)$ & $\mathrm{b}\left(\mathrm{g} \times 10^{-4}\right)$ & $\mathrm{c}\left(\mathrm{g} \times 10^{-5} \mathrm{~h}^{-1}\right)$ & $\mathrm{T}_{1 / 2}(\mathrm{~h})$ & $\mathrm{T}_{(\mathrm{a}+\mathrm{b})}(\mathrm{h})$ \\
\hline $2.5(1)$ & $13 \pm 23^{\mathrm{a}}$ & $47.93 \pm 5.23^{\mathrm{a}}$ & $7325 \pm 2.2^{\mathrm{a}}$ & $9.46 \pm 0.0027^{\mathrm{a}}$ & $192^{\mathrm{a}}$ \\
$1.25(2)$ & $5 \pm 9^{\mathrm{a}}$ & $48.09 \pm 6.27^{\mathrm{a}}$ & $7194 \pm 249^{\mathrm{a}}$ & $9.64 \pm 0.34^{\mathrm{a}}$ & $192^{\mathrm{a}}$ \\
$5(1)$ & $38 \pm 50^{\mathrm{a}}$ & $98.88 \pm 4.64^{\mathrm{b}}$ & $6720 \pm 990^{\mathrm{a}}$ & $10.52 \pm 1.81^{\mathrm{a}}$ & $204^{\mathrm{a}}$ \\
$2.5(2)$ & $12 \pm 24^{\mathrm{a}}$ & $95.57 \pm 15.99^{\mathrm{b}}$ & $6666 \pm 115^{\mathrm{a}}$ & $10.69 \pm 2.23^{\mathrm{a}}$ & $216^{\mathrm{a}}$ \\
\hline
\end{tabular}

a: Excreción inicial en el tiempo cero de $\mathrm{TiO}_{2} ;$ b: Excreción potencial de $\mathrm{TiO}_{2} ; \mathrm{c}$ : Tasa de excreción de $\mathrm{TiO}_{2} ; \mathrm{T}_{1 / 2}$ : Tiempo necesario de excreción de $\mathrm{TiO}_{2}$ para que haya excretado la mitad de la concentración inicial; $\mathrm{T}_{(\mathrm{a}+\mathrm{b})}$ : Tiempo en el que se alcanzó el equilibrio constante de $\mathrm{TiO}_{2}$

${ }^{a, b}$ Superíndices diferentes dentro de columnas indican diferencia estadística $(p<0.0001)$

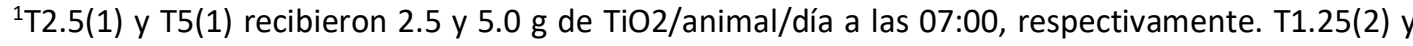

T2.5(2) recibieron 2.5 y $5.0 \mathrm{~g}$ de TiO2/animal/día distribuido en dos dosis a las 07:00 y 16:00

Cuadro 3. Producción (promedio $\pm \mathrm{DE}$ ) de heces estimada con dióxido de titanio $\left(\mathrm{TiO}_{2}\right)$ y observada mediante bolsas colectoras en alpacas

\begin{tabular}{cccc}
\hline Tratamiento $^{1}$ & Producción estimada $(\mathrm{g})$ & Producción observada $(\mathrm{g})$ & Probabilidad \\
\hline $2.5(1)$ & $533.1 \pm 71.3$ & $469.5 \pm 74.1$ & 0.127710 \\
$1.25(2)$ & $543.9 \pm 97.2$ & $413.3 \pm 99.3$ & 0.001214 \\
$5(1)$ & $537.2 \pm 54.5$ & $445.6 \pm 29.1$ & 0.036063 \\
$2.5(2)$ & $679.9 \pm 115.0$ & $344.8 \pm 73.7$ & 0.000030 \\
\hline
\end{tabular}

${ }^{1} \mathrm{~T} 2.5(1)$ y $\mathrm{T} 5(1)$ recibieron 2.5 y $5.0 \mathrm{~g}$ de TiO2/animal/día a las 07:00, respectivamente. T1.25(2) y

T2.5(2) recibieron 2.5 y $5.0 \mathrm{~g}$ de TiO2/animal/día distribuido en dos dosis a las 07:00 y 16:00 
Cuadro 4. Ecuación de la regresión lineal entre la producción de heces estimada con dióxido de titanio (X) y la observada (Y) en alpacas

\begin{tabular}{ccc}
\hline Tratamiento $^{1}$ & Regresión lineal & $\mathrm{R}^{2}$ \\
\hline $2.5(1)$ & $\mathrm{y}=0.5981 \mathrm{x}+150.67$ & 0.3318 \\
$1.25(2)$ & $\mathrm{y}=0.411 \mathrm{x}+222.08$ & 0.5912 \\
$5(1)$ & $\mathrm{y}=0.041 \mathrm{x}+391.23$ & 0.0016 \\
$2.5(2)$ & $\mathrm{y}=0.4997 \mathrm{x}+5.1584$ & 0.6083 \\
\hline
\end{tabular}

${ }^{1} \mathrm{~T} 2.5(1)$ y T5(1) recibieron 2.5 y $5.0 \mathrm{~g}$ de TiO2/animal/día a las 07:00, respectivamente. T1.25(2) y $\mathrm{T} 2.5(2)$ recibieron 2.5 y $5.0 \mathrm{~g}$ de TiO2/animal/día distribuido en dos dosis a las 07:00 y 16:00

(Owens y Hanson, 1992). En el presente estudio, el $\mathrm{TiO}_{2}$ en alpacas no estaría cumpliendo este criterio, ya que la mayor recuperación fue del $90 \%$ en promedio parra T2.5(1). Sin embargo, si la recuperación fecal del marcador es diferente de $100 \%$ podría ser usado como tal si es que la diferencia es constante.

\section{Patrón de Excreción de $\mathrm{TiO}_{2}$}

Los promedios de los parámetros del patrón de excreción del $\mathrm{TiO}_{2}$ en alpacas se presentan en el Cuadro 2. Se observa que la excreción inicial del $\mathrm{TiO}_{2}$ en el tiempo cero (a), la tasa constante de excreción de b (c), el tiempo necesario de excreción de $\mathrm{TiO}_{2}$ para que haya excretado la mitad de la concentración inicial $\left(\mathrm{T}_{1 / 2}\right)$ y el tiempo en el que se alcanza el equilibrio de excreción del marcador $\left(\mathrm{T}_{(\mathrm{a}+\mathrm{b})}\right)$ no fueron estadísticamente diferentes entre tratamientos. La máxima excreción de $\mathrm{TiO}_{2}$ (b) fue significativamente menor en los tratamientos que recibieron menor dosis [T2.5(1), T1.25(2)] en comparación con aquellos que recibieron mayor dosis [T5(1), T2.5(2)] $(\mathrm{p}<0.05)$.

El tiempo medio fue aproximadamente a las $10.08 \mathrm{~h}$, tiempo en el que se excretó la mitad de la concentración del $\mathrm{TiO}_{2}$ y el tiempo en que el marcador se alcanza el equilibrio constante de excreción fue a las $201 \mathrm{~h}$
(8 días en promedio) después del inicio de la administración del $\mathrm{TiO}_{2}$. Este resultado difiere del trabajo de Glindemann (2007) con ovejas, quien observa que el tiempo para lograr el equilibrio de excreción del $\mathrm{TiO}_{2}$ fue de cinco días. Sin embargo, el mayor tiempo obtenido en el presente estudio podría deberse a la lenta tasa de retención del alimento en el tracto digestivo de los CSA, una de las particularidades de esta especie (San Martín y Van Saun, 2014).

Le Du y Penning (1982) atribuyen el tiempo en que se alcanza la excreción constante del marcador en las heces a la calidad de las pasturas, donde alimentos más fibrosos registran tiempos más largos de excreción a diferencia de pastos de buena calidad o concentrados. Además, cuanto mayor es el nivel de ingesta del alimento, la excreción del marcador sería ligeramente más rápida $\mathrm{y}$, como se sabe, los camélidos presentan menor consumo de alimento que los ovinos (San Martín y Bryant, 1987). Por lo tanto, esto podría explicar el tiempo en el que se alcanza la excreción constante del $\mathrm{TiO}_{2}$ en el presente estudio.

Los parámetros del patrón de excreción de $\mathrm{TiO}_{2}$ no estuvieron influenciados por la mayor frecuencia de administración del marcador. Sin embargo, algunos autores describen que existe un típico patrón diurno de ex- 
creción del $\mathrm{TiO}_{2}$ (Hafez et al., 1998; Glindemann, 2007). Prigge et al. (1981) observaron variación diurna del patrón de excreción cuando las vacas fueron dosificadas dos veces al día. Así mismo, Myers et al. (2006) indican que no encontraron variaciones diurnas notables en el patrón de excreción fecal para el $\mathrm{TiO}_{2}$, posiblemente por haber dosificado dos veces por día.

\section{Producción de Heces con $\mathrm{TiO}_{2}$}

Se encontraron diferencias significativas entre los tratamientos T1.25(2), T5(1) y T2.5(2) entre la producción estimada vs. la producción observada en las bolsas colectoras ( $<<0.05$; Cuadro 3). La producción de heces fue similar únicamente en el tratamiento T2.5(1). Titgemeyer et al. (2001), por otro lado, solo encuentra valores numéricamente mayores de la producción de heces estimada con el uso de $\mathrm{TiO}_{2}$ versus la colección de heces total, pero sin observarse diferencia significativa.

En el presente estudio, el porcentaje de sobreestimación fue de 13, 32, 20 y $97 \%$ para T2.5(1), T1.25(2), T5(1) y T2.5(2), respectivamente, a diferencia de lo reportado por De Souza et al. (2015) quiénes obtuvieron 4\% de sobreestimación, llegando a la conclusión que el $\mathrm{TiO}_{2}$ en vacas lecheras estima valores de producción de excreción fecal con gran precisión. Por el contrario, Glindemann (2007) encontró subestimaciones de la producción de heces en ovejas, atribuyendo sus resultados a una recuperación por encima del 100\% del $\mathrm{TiO}_{2}$. El momento en que se toma la muestra es uno de los principales problemas para estimar con mejor precisión la producción fecal, debido la variación diurna de la excreción fecal del marcador (Dove y Mayes, 1991).

Las ecuaciones de regresión lineal entre la producción de heces estimada con $\mathrm{TiO}_{2}$ y producción observada directa de heces en alpacas se presentan el Cuadro 4. Se observa que los tratamientos T1.25(2) y T2.5(2) presentaron los mayores coeficientes de correlación; resultados que coinciden con el trabajo de Glindemann (2007) en ovejas que recibieron $\mathrm{TiO}_{2}$ dos veces al día. En la ecuación de la regresión lineal del T2.5(2) se observa que el valor de b (5.1584) es el más cercano a cero a diferencia de los otros tratamientos, por lo tanto, es el modelo que presenta mejor ajuste entre las variables.

No se reportan estudios previos que consideren aspectos relacionados con el uso de algún marcador nutricional en CSA, así como lo referente a sus similitudes o diferencias con rumiantes como el ovino o el vacuno; por lo tanto, este podría ser uno de los primeros ensayos utilizando el $\mathrm{TiO}_{2}$ como marcador para estimar producción de heces en alpacas.

Las características anatomo-fisiológicas particulares de la alpaca, tales como su tasa de retención lenta del alimento en el tracto digestivo, su alta digestibilidad de alimentos de baja calidad, su alta capacidad de reciclaje de nitrógeno, su menor requerimiento nutricional que le permiten sobrevivir en un estado de homeostasis en ambientes de escasez de alimento, su menor consumo de alimento, su comportamiento altamente selectivo y su capacidad de adaptación a las praderas alto-andinas, caracterizadas por su baja disponibilidad de forraje y cuyos nutrientes están altamente diluidos por carbohidratos estructurales de difícil digestión (Van Saun, 2003; San Martín y Van Saun, 2014) podrían estar influyendo en la variabilidad de los resultados obtenidos en el presente estudio.

El tiempo medio de $10.08 \mathrm{~h}$ de excreción del $\mathrm{TiO}_{2}$ concuerda con otros reportes en ovejas (Myers et al., 2006; Glindemann, 2007). Sin embargo, el tiempo en que se alcanza el equilibrio de excreción del marcador fue cercano a 8 días, mayor a los 5 días reportado por Glindemann (2007), posiblemente debido a la tasa de retención lenta del alimento en el tracto digestivo de los CSA (San Martín y Van Saun, 2014). 


\section{ConClusiones}

- La recuperación del $\mathrm{TiO}_{2}$ en heces de alpacas administradas con $2.5 \mathrm{~g} /$ día, 1.25 $\mathrm{g}$ dos veces al día, $5 \mathrm{~g}$ /día y $2.5 \mathrm{~g}$ dos veces al día fueron de $90,82,78$ y $52 \%$.

- La excreción potencial del $\mathrm{TiO}_{2}$ en tiempo $t$ (valor de b), es influenciado directamente por las cantidades administradas del marcador.

- El tiempo medio $\left(\mathrm{T}_{1 / 2}\right)$ de la excreción de $\mathrm{TiO}_{2}$ se alcanza a las $10.08 \mathrm{~h}$ de la administración del marcador.

- El tiempo que alcanza el equilibrio de excreción del $\mathrm{TiO}_{2}$ es aproximadamente a los 8 días.

- La cantidad de $2.5 \mathrm{~g}$ de $\mathrm{TiO}_{2} /$ animal, administradas una vez al día, estima valores similares de producción de heces a la medida con las bolsas colectoras.

- El uso de $\mathrm{TiO}_{2}$ como marcador externo para estimar producción de heces es factible en alpacas.

\section{Literatura Citada}

1. Barton NWH, Houston DC. 1991. The use of titanium dioxide as an inert marker for digestion studies in raptors. Comp Biochem Phys A 100: 1025-1029. doi: 10.1016/0300-9629(91)90331-6

2. De Souza J, Batistel F, Welter KC, Silva MM, Costa DF, Portela FA. 2015. Evaluation of external markers to estimate fecal excretion, intake, and digestibility in dairy cows. Trop Anim Health Pro 47: 265-268. doi: 10.1007/ s11250-014-0674-6

3. Dove H, Mayes RW. 1991. The use of plant wax alkanes as marker substances in studies of the nutrition of herbivores: a review. Aust J Agr Res 42: 913-952. doi: 10.1071/AR9910913

4. Forbes JM, Kyriazakis I. 1995. Food preferences in farm animals: why don't they always choose wisely? P Nutr Soc 54: 429-440. doi: 10.1079/PNS19950012
5. Glindemann T. 2007. Effect of grazing intensity on feed intake and productivity of sheep in the Inner Mongolian steppe. PhD Thesis. Kiel, Germany: ChristianAlbrechts Universität zu Kiel. p 117.

6. Glindemann T, Tas BM, Wang $C$, Alvers S, Susenbeth, A. 2009. Evaluation of titanium dioxide as an inert marker for estimating faecal excretion in grazing sheep. Anim Feed Sci Tech 152: 186-197. doi: 10.1016/ j.anifeedsci.2009.04.010

7. Greenhalgh, JFD. 1982. An introduction to herbage intake measurements. In: Leaver JD (ed). Herbage intake handbook. UK: The British Grassland Society. p 1-9.

8. Hafez S, Junge W, Kalm E. 1988. Estimate of digestibility in dairy cows using an indicator method in comparison to the Hohenheimer feed value test. Arch Tierernahr 38: 929-945. doi: 10.1080/ 17450398809430920

9. Haro JM. 2012. Consumo voluntario de forraje por rumiantes en pastoreo. Acta Universitaria 12: 56-63. doi: 10.15174/ au.2002.283

10. Hellwing ALF, Lund P, Weisbjerg MR, Oudshoorn FW, Munksgaard L, Kristensen T. 2015. Comparison of methods for estimating herbage intake in grazing dairy cows. Livestock Sci 176: 61-74. doi: 10.1016/j.livsci.2015.01.013

11. Hodgson J. 2004. Measurements of herbage intake and ingestive behaviour in grazing animals: an introduction. In: Penning $P$ (ed). Herbage intake Handbook. UK: British Grassland Society. p 15-21.

12. Le Du YLP, Penning PD. 1982. Animal based techniques for estimating herbage intake. In: Leaver JD (ed). Herbage intake handbook. UK: British Grassland Institute. p 37-75.

13. Mayes $R W$, Dove $H .2000$. Measurement of dietary nutrient intake in free-ranging mammalian herbivores. Nutr Res Rev 13: 107-138. doi: 10.1079/ 095442200108729025 
14. Mayland HF, Florence AR, Rosenau RC, Lazar VA, Turner HA. 1975. Soil ingestion by cattle on semiarid range as reflected by titanium analysis of feces. $\mathrm{J}$ Range Manage 28: 448-452. doi: $10.2307 / 3897219$

15. McDonald E, Greenhalgh M. 1999. Nutrición animal. $5^{\text {th }}$ ed. Zaragoza, España: Acribia. $600 \mathrm{p}$.

16. [MINAGRI] Ministerio de Agricultura y Riego. 2017. Perú es el mayor productor de fibra de alpaca en el mundo. [Internet]. Disponible en: http:// www.minagri.gob.pe/portal/notas-2017/ 19720-peru-es-el-mayor-productor-defibra-de-alpaca-en-el-mundo

17. Myers WD, Ludden PA, Nayigihugu $V$, Hess BW. 2004. A procedure for the preparation and quantitative analysis of samples for titanium dioxide. J Anim Sci 82: 179-183.

18. Myers WD, Ludden PA, Nayigihugu $V$, Hess $B W$. 2006. Excretion patterns of titanium dioxide and chromic oxide in duodenal digesta and feces of ewes. Small Ruminant Res 63: 135-141. doi: 10.1016/j.smallrumres.2005.02.010.

19. Njaa LR. 1961. Determination of protein digestibility with titanium dioxide as indicator substance. Acta Agr Scand 11: 227-241. doi: 10.1080/00015126109434994

20. Ohmori H, Nonaka I, Ohtani F, Tajima K, Kawashima T, Kaji Y, Terada F. 2013. An improved dry ash procedure for the detection of titanium dioxide in cattle feces. Anim Sci J 84: 726-731. doi: 10.1111/asj.12068.

21. Orskov ER, McDonald I. 1979. The estimation of protein degradability in the rumen from incubation measurements weighted according to rate of passage. J Agr Sci-Cambridge 92: 499-503. doi: 10.1017/S0021859600063048.

22. Owens FN, Hanson CF. 1992. External and internal markers for appraising site and extent of digestion in ruminants. J Dairy Sci 75: 2605-2617. doi: 10.3168/jds.S0022-0302(92)78023-0

23. Peddie J, Dewar WA, Gilbert AB, Waddington D. 1982. The use of titanium dioxide for determining apparent digestibility in mature domestic fowls (Gallus domesticus). J Agr Sci 99: 233236. doi: 10.1017/S002185960005526X

24. Prigge EC, Varga GA, Vicini JL, Reid RL. 1981. Comparison of ytterbium chloride and chromium sesquioxide as fecal indicators. J Anim Sci 53: 16291633. doi: $10.2527 /$ jas $1982.5361629 \mathrm{x}$

25. San Martín F, Van Saun RJ. 2014. Applied digestive anatomy and feeding behavior. In: Cebra C, Anderson D, Tibary A, Van Saun R, Johnson LR (eds). Llama and alpaca care: medicine, surgery, reproduction, nutrition, and herd health. Canada: Elsevier. p 51-58.

26. Titgemeyer EC. 1997. Design and interpretation of nutrient digestion studies. J Anim Sci 75: 2235-2247. doi: $10.2527 / 1997.7582235 \mathrm{x}$

27. Titgemeyer EC, Armendariz CK, Bindel DJ, Greenwood RH, Löest CA. 2001. Evaluation of titanium dioxide as a digestibility marker for cattle. J Anim Sci 79: 1059-1063. doi: 10.2527/ 2001.7941059x

28. Van Saun RJ. 2003. Feeding the alpaca. In: Hoffman E (ed). The complete alpaca book. California, USA: Bonny Doon Press. p 179-232. 